\title{
Serotonin transporter polymorphism (5HTTLPR), severe childhood abuse and depressive symptom trajectories in adulthood
}

Timothy B. Nguyen, Jane M. Gunn, Maria Potiriadis, Ian P. Everall and Chad A. Bousman

\section{Background}

Cross-sectional studies suggest that the serotonin transporter promoter region polymorphism (5-HTT genelinked polymorphic region, 5HTTLPR) moderates the relationship between childhood abuse and major depressive disorder.

\section{Aims \\ To examine whether the $5 H T T L P R$ polymorphism moderates the effect childhood abuse has on 5-year depressive symptom severity trajectories in adulthood. \\ Method \\ At 5-year follow-up, DNA from 333 adult primary care attendees was obtained and genotyped for the 5HTTLPR polymorphism. Linear mixed models were used to test for a genotype $\times$ childhood abuse interaction effect on 5-year depressive symptom severity trajectories.}

\section{Results}

After covariate adjustment, homozygous $s$ allele carriers with a history of severe childhood abuse had significantly greater depressive symptom severity at baseline compared with those without a history of severe childhood abuse and this effect persisted throughout the 5-year period of observation.

\section{Conclusions}

The $5 H T T L P R$ S/S genotype robustly moderates the effects of severe childhood abuse on depressive symptom severity trajectories in adulthood.

\section{Declaration of interest}

None.

\section{Copyright and usage}

(c) The Royal College of Psychiatrists 2015. This is an open access article distributed under the terms of the Creative Commons Non-Commercial, No Derivatives (CC BY-NC-ND) licence.
Exposure to adverse life events during childhood has consistently been linked to increased risk of depression in adulthood. ${ }^{1,2}$ However, early life exposure to adversity alone is likely not sufficient to elicit depression later in life. In fact, evidence to date suggests that some individuals are more susceptible to negative outcomes following exposure to adversity and that this susceptibility in part may reside in an individual's genome.

Over the past decade, genetic variation in over 35 genes have been examined for their interaction with adverse life events. ${ }^{3}$ Among these genes, the serotonin transporter (SLC6A4) and its corresponding length polymorphisms located in the $5^{\prime}$-flanking promoter region (5-HTT gene-linked polymorphic region, $5 H T T L P R)^{4}$ is arguably the most studied in relation to depression and exposure to adverse life events, namely severe childhood abuse. The initial study conducted by Caspi et $a l^{5}$ reported that young adults with the $s$ allele were more likely to develop depression after exposure to severe childhood abuse compared with those with the $l$ allele and proposed that the $s$ allele may be a moderator (effect modifier) of the relationship between stress and depression. However, meta-analyses have both supported ${ }^{6}$ and rejected this notion ${ }^{7}$ and researchers have since criticised the reliance on cross-sectional designs, inadequate adjustment for potential confounders and clinical utility of the gene $\times$ environment studies conducted to date. ${ }^{8,9}$

In response to these criticisms, the present study examined the interaction between the 5HTTLPR polymorphism and history of severe childhood abuse on depressive symptom trajectories in a 5 -year prospective cohort of primary care attendees. Linear mixed modelling was used to test whether 5HTTLPR genotype moderated the effect of severe childhood abuse on depressive symptom trajectories. We hypothesised that $s / s$ carriers would be more likely to experience severe depressive symptom trajectories following exposure to severe childhood abuse compared with $s / l$ or $l / l$ carriers.

\section{Method}

\section{Participants}

Participants were recruited from the Diagnosis, Management and Outcomes of Depression in Primary Care (diamond) study, an ongoing prospective cohort that commenced in 2005 with an aim to document the experiences, health outcomes, treatment and service use of primary care patients identified as having clinically relevant depressed mood at screening from 30 rural and metropolitan general practices randomly recruited in Victoria, Australia. ${ }^{10}$ Patients were eligible for the diamond cohort if they were: (a) aged 18-75 years, (b) able to read English, (c) not terminally ill, (d) did not reside in a nursing home and (e) scored 16 or higher on the Center for Epidemiologic Studies Depression Scale. ${ }^{11}$ Participants were assessed annually using postal surveys as well as computer-assisted telephone interviews. In 2011 (cohort year 6), participants enrolled in the cohort were invited to provide a saliva sample for DNA extraction and genotyping.

All procedures were conducted in accordance with principles expressed in the Declaration of Helsinki and obtained approval from the University of Melbourne Human Research Ethics Committee (Ethics ID 1135247.1).

\section{Measures}

The main outcome of interest was depression symptom severity assessed at baseline and 1-, 2-, 3-, 4- and 5-year post-baseline using the Primary Care Evaluation of Mental Disorders Patient Health Questionnaire-9 (PHQ-9). The PHQ-9 is a self-administered questionnaire of depressive symptoms based directly on the nine 
signs and symptoms of major depressive disorder as described in the DSM-IV ${ }^{12}$ and has been validated to screen and monitor depression severity in the primary care setting. ${ }^{13}$ The PHQ-9 asks respondents to rate their symptoms over the past 2 weeks and is scored on a scale of 0 ('not at all') to 3 ('nearly every day') for each item with a range of $0-27 .{ }^{14}$ Scores of 5, 10, 15 and 20 on the PHQ-9 represent cut-points for mild, moderate, moderately severe and severe depression respectively. ${ }^{14}$

The Child Maltreatment History Self-Report (CMHSR) ${ }^{15}$ was used to measure history of severe childhood abuse. The CMHSR includes questions relating to sexual and physical abuse by an adult before 16 years of age. The distinction of severe physical abuse was originally based on expert consensus that responses in this severe category were more likely to cause injury requiring medical treatment and has been shown to have replicable psychometric properties. ${ }^{16}$ The distinction of severe sexual abuse was based on items from a national Canadian survey of unwanted touching in sexual areas and attempted or achieved unwanted intercourse. ${ }^{17}$

At baseline, assessments were also made of age, gender highest level of completed education, smoking status, family history of depression, present DSM-IV diagnosis of depression, antidepressant use, anxiolytic use, herbal/alternative medication use, health status, ${ }^{18}$ quality of life, ${ }^{19}$ alcohol abuse/dependence ${ }^{20}$ and significant negative life events in the past year. ${ }^{21}$

\section{Polymorphism selection, DNA extraction and genotyping}

The 5HTTLPR and its A-to-G internal single nucleotide polymorphism (rs25531) were selected for genotyping. Individuals were categorised as $s / s$ if they were $s / s, s / l_{G}$ or $l_{G} / l_{G}$. Those who had $s / l_{A}$ or $l_{G} / l_{A}$ were categorised as $s / l$ and individuals with $l_{\mathrm{A}} l_{\mathrm{A}}$ were categorised as $l / l$. To detect the presence of population stratification, 60 unlinked ancestry informative markers (AIMs; Table DS1) representing the three HapMap phase III populations (Northern/Western European, Han Chinese and Yoruba in Nigeria) were also genotyped. ${ }^{22}$

DNA was recovered from stabilised saliva samples using the manual prep IT system according to manufacturer's instructions (Oragene DNA (OG-500); DNA Genotek, Ontario, Canada). DNA precipitates were allowed to resuspend for a minimum of $48 \mathrm{~h}$ before quantification by fluorimetry (QuantiFluor ${ }^{\mathrm{TM}} \mathrm{dsDNA}$ System; Promega Corporation, Madison, Wisconsin, USA) in conjunction with a Gemini ${ }^{\mathrm{TM}}$ Spectramax XPS fluorescence microplate reader (Molecular Devices, LLC, Sunnyvale, California, USA). DNA stocks were adjusted to a working concentration of between 10 and $50 \mathrm{ng} / \mu \mathrm{L}$ for subsequent genotyping.

The SLC6A4 locus was amplified for both the 5HTTLPR and rs25531 polymorphisms. The promoter region was amplified using the primers SERT1 and SERT2. ${ }^{23}$ The 5HTTLPR polymorphism was analysed as described in Wendland et $a l^{24}$ with modifications. Reactions were performed in a total volume of $24 \mu \mathrm{L}$ comprising 'KapaTaq A' polymerase (0.6 units), 'High-Yield' Reaction Buffer A $(2.4 \mu \mathrm{L})$; both Kapa Biosystems, Boston, Massachusetts, USA), $0.48 \mu \mathrm{L}$ dNTP mix $(2.5 \mathrm{mM}$; Bioline Australia, Alexandria, New South Wales, Australia), 20-50 ng template DNA, and supplementary $\mathrm{Mg}^{2+}$ (1.0 mM final; $\mathrm{MgSO}_{4}$, $50 \mathrm{mM}$; Bioline Australia) and 7-deaza-dGTP (0.2 mM final; New England Biolabs, Ipswich, Massachusetts). After initial denaturation $\left(95^{\circ} \mathrm{C}, 7 \mathrm{~min}\right)$, cycling parameters were $94^{\circ} \mathrm{C}(60 \mathrm{~s})$, $61^{\circ} \mathrm{C}(60 \mathrm{~s}), 72^{\circ} \mathrm{C}(60 \mathrm{~s})(20 \mathrm{cycles})$ followed by a further 20 cycles of $94^{\circ} \mathrm{C}(60 \mathrm{~s}), 63^{\circ} \mathrm{C}(60 \mathrm{~s}), 72^{\circ} \mathrm{C}(60 \mathrm{~s})$, and a final extension period of $72^{\circ} \mathrm{C}$ for $30 \mathrm{~min}$. All polymerase chain reactions were conducted using Veriti 96-well thermal cyclers (Life Technologies Australia Scoresby, Victoria, Australia). Genotypes were determined by capillary electrophoresis using an AB3730 Genetic Analyser fitted with a $36 \mathrm{~cm}$ array, with sizing determined against a Genescan LIZ500 molecular weight marker. Analysis was performed in Genemapper V3.7 software (Life Technologies Australia, Scoresby, Victoria, Australia).

\section{Statistical analysis}

Chi-squared analysis was used to detect departures from HardyWeinberg equilibrium of 5HTTLPR. To estimate the presence of population stratification, the 60 AIMs were used to assign each participant to the HapMap ancestral group for which they carried the greatest proportion of that population's AIMs.

Linear mixed models were used to determine trajectory differences in PHQ-9 depressive symptom severity over the 5 -year follow-up period by 5 HTTLPR and history of severe child abuse. Before modelling, genotype variables and covariates were centred. ${ }^{25}$ Potential covariates were assessed for their association with abuse history and genotype using chi-squared, Fisher's exact or analysis of variance tests, depending on the variable structure. Covariates with $P$-values $\leq 0.05$ were retained for adjusted analyses (Table 1). The unadjusted model included fixed effects of time, genotype, child abuse, and a time $\times$ genotype, time $\times$ child abuse, genotype $\times$ child abuse, and time $\times$ genotype $\times$ child abuse interaction terms as well as random effects of individual, primary care site, intercept (baseline PHQ) and time. Adjusted models included relevant covariates as well as covariate $\times$ time, covariate $\times$ genotype, and covariate $\times$ child abuse interaction terms as recently recommended. ${ }^{26}$ Covariance models used for the random and repeated effects were unstructured and first-order autoregressive, respectively. To improve model fit, a stepwise elimination strategy was employed by comparing -2 log likelihood ( $-2 \mathrm{LL}$ ) information criteria using unrestricted maximum likelihood estimations. The covariate term with the lowest $F$-test score was removed, one at a time, and discarded if the difference in the $-2 \mathrm{LL}$ between the simpler model and more complex model was less than 3.86 (i.e. chi-squared value with 1 degree of freedom at $P=0.05)$. The final reduced adjusted model was tested with restricted maximum likelihood estimation. Modelling was performed using the SPSS software (SPSS, version 22.0, IBM, Chicago, Illinois, USA). ${ }^{27}$

\section{Results}

A total of 498 individuals were enrolled in the diamond study at the time of DNA collection in cohort year 6 and 344 (69\%) participants returned a DNA sample. Six individuals who were missing genotype data and five individuals who were missing childhood abuse data were excluded, resulting in a total of 333 individuals available for analysis (Table 1). No differences in demographic or clinical characteristics were detected by genotype. However, sedative use, self-rated health, non-alcohol-related substance abuse and the abbreviated World Health Organization Quality of Life and Functioning instrument of social relationships (WHOQOL-BREF social) differed by history of severe childhood abuse (Table 1) and as such were included in the adjusted models. 5HTTLPR genotype was in Hardy-Weinberg equilibrium $\left(\chi^{2}=3.51, P \geq 0.06\right)$ and all participants were of Northern/Western European ancestry based on 60 unlinked AIMs.

The effect of severe child abuse on depressive symptoms trajectories was moderated by 5HTTLPR genotype $\left(F_{2,318.2}=3.1\right.$, $p_{\text {covariate adjusted }}=0.047$ ). Post hoc analysis (Fig. 1) showed that $s / s$ genotype carriers with a history of child abuse had significantly greater baseline depressive symptom severity compared with those without a history (mean difference $=3.4,95 \%$ CI 1.2-5.6, $p_{\text {covariate adjusted }}=0.003$ ) and this effect persisted throughout the 


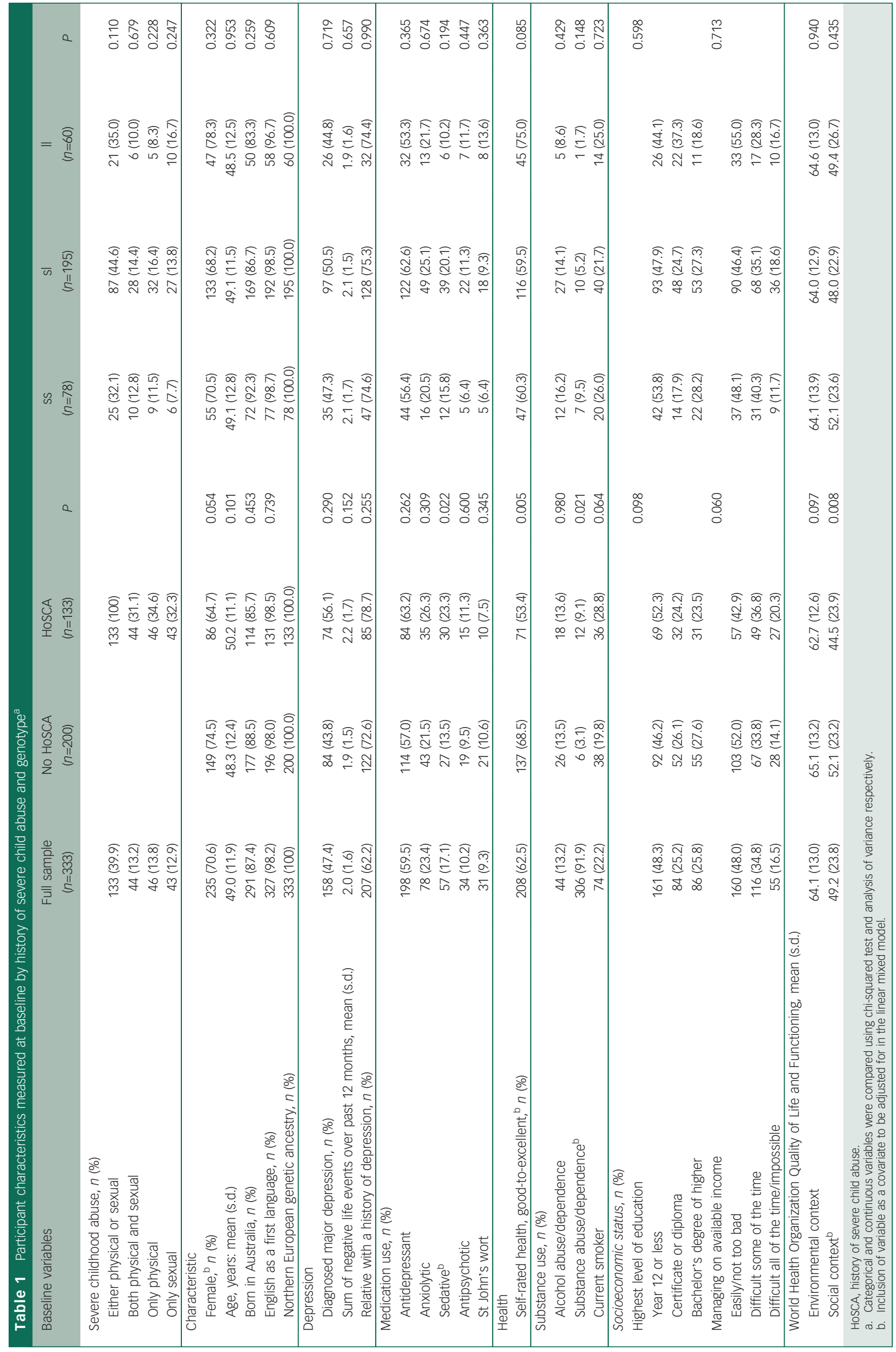




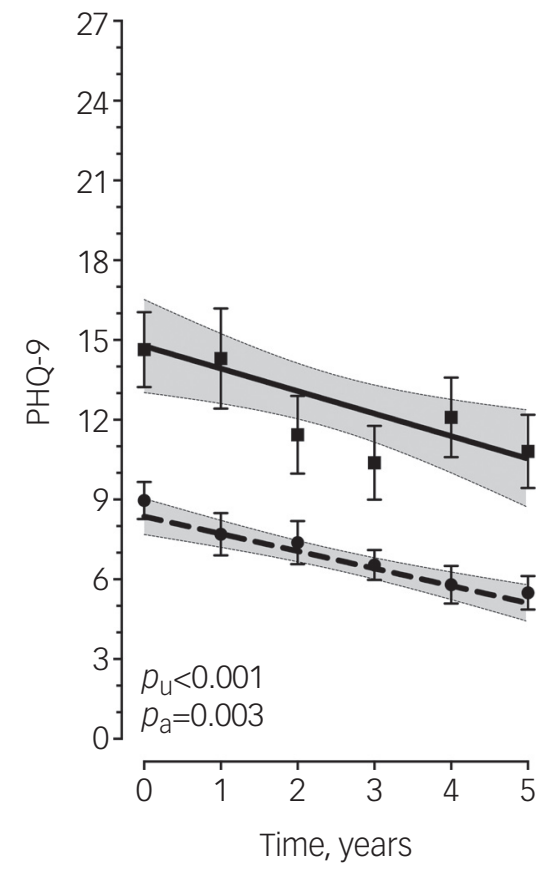

$\mathrm{Sl}$

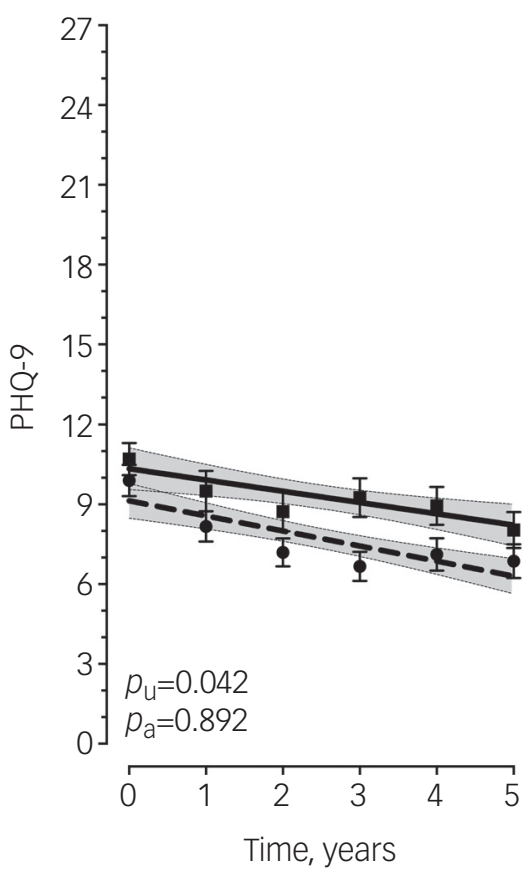

॥

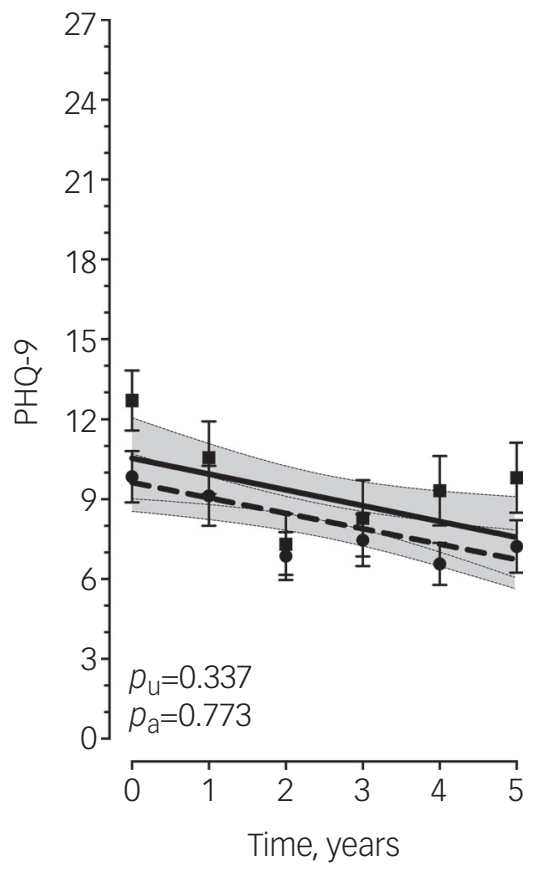

Fig. 1 Longitudinal measurements of depressive symptoms over 5 years by 5 HTTLPR and history of severe child abuse.

Points are observed mean PHQ-9 scores with standard error bars. Lines are predicted values with $95 \%$ confidence intervals shaded based on linear mixed model analysis. Dashed line/ circles, no history of severe child abuse; solid line/squares, history of severe child abuse; PHQ-9, Primary Care Evaluation of Mental Disorders Patient Health Questionnaire-9; circles, no history of severe child abuse; solid line/squares, history of severe child abuse; PHQ-9, Primary
$p_{\mathrm{U}}$, covariate-unadjusted $P$-value; $p_{\mathrm{A}}$, covariate-adjusted $P$-value. $P$-values based on estimated marginal means.

5 -year period of observation. However, individuals with the $l / s$ or l/l genotype had similar depressive symptom trajectories regardless of severe child abuse history $(s / l$ : mean difference $=0.8$, $95 \%$ CI $-1.1-1.3, p_{\text {covariate adjusted }}=0.892 ; \mathrm{l} / \mathrm{l}$ : mean difference $=0.3$, 95\% CI $-1.9-2.5, p_{\text {covariate adjusted }}=0.773$ ). Furthermore, among individuals without a history of severe child abuse, $s / s$ genotype carriers appeared to have more favourable depressive symptom trajectories compared with $s / l$ and $l / l$ genotype carriers, albeit not statistically significant $\left(F=2.72, p_{\text {covariate adjusted }}=0.068\right.$, Fig. DS1). Parameter estimates for all terms included in the unadjusted and adjusted models are provided in Tables DS2 and DS3 respectively.

\section{Discussion}

In accordance with our hypothesis and previous cross-sectional studies, ${ }^{6}$ we observed greater depressive symptom severity among individuals with the $s / s$ genotype who reported a history of severe child abuse compared with those without a history. We also provide novel evidence which suggests that the $s / s$ genotype confers a persistent moderating effect on depressive symptom severity without alteration to the rate of change over time. There was no significant difference in depressive symptoms between those with or without a history of child abuse in the $s / l$ or $l / l$ genotype groups. These findings support the notion that long-lasting consequences of adverse childhood experiences may be dependent on an individual's genomic context. ${ }^{28}$ More specifically, these findings suggest that the $s / s$ genotype may be associated with susceptibility to more severe depressive symptom trajectories in adulthood following exposure to severe abuse in childhood.

Among the $s / s$ group, those with a history of severe, child abuse had a mean PHQ-9 score greater than 10 (i.e. moderate depression) at each time point over the 5-year period, whereas those without a history of child abuse consistently scored under 10 (i.e. mild depression), a differential effect not observed among $s / l$ and $l / l$ genotype carriers. However, among individuals without a history of severe child abuse, we detected a trend in which $s / s$ genotype carriers had more favourable depressive symptom trajectories compared with $s / l$ and $l / l$ genotype carriers. This pattern of results suggests that the 5HTTLPR polymorphism may be a marker of 'phenotypic plasticity' rather than 'vulnerability' in that $s / s$ carriers appear to be the most susceptible to the negative effects of severe child abuse (i.e. increased depressive symptom severity) but also marginally more likely to benefit from the absence of a severe child abuse experience, albeit only a trend difference $(P=0.068)$ was seen between $s / s$ carriers and their $l / l$ or $l / s$ carrying counterparts in the absences of severe child abuse. Nonetheless, this so-called "differential susceptibility ${ }^{29}$ has been observed previously. Most notably, in the landmark paper by Caspi $e t a l^{5}$ in that $s / s$ genotype carriers with stressful life events reported the most depressive symptoms but conversely those without such events reported the fewest depressive symptoms. Similar results for the $s / s$ genotype have been observed more recently. Eley et $a l^{30}$ showed the $s / s$ genotype conferred more susceptibility to depression among female adolescents in highstress environments but $s / s$ genotype carriers in low-stress environments were less likely to be depressed. Furthermore, Wilhelm et $a l^{31}$ showed that the probability of lifetime major depression was highest among $s / s$ carriers with more than one adverse life event in the past 5 years but lowest for $s / s$ carriers with no such events.

If the 5HTTLPR polymorphism is a marker of "phenotypic plasticity', it may have clinical utility in guiding psychosocial intervention in the future. In fact, recent work showed that children with anxiety with the $s / s$ genotype were more likely to 
respond to cognitive-behavioural therapy compared with $s / l$ and $l / l$ genotypes, ${ }^{32}$ albeit this was not observed in a randomised control trial of cognitive-behavioural therapy among adults with recurrent depression. ${ }^{33}$ Furthermore, the antidepressant pharmacogenomics literature suggests that, among White but not Asian people, the $l / l$ rather than the $s / s$ genotype infers 'phenotypic plasticity' in that $s$ allele carriers are less likely to respond to selective serotonin reuptake inhibitors compared with individuals with the $l / l$ genotype. ${ }^{34}$ Likewise, there is evidence, primarily in adolescent studies, suggesting the $s / s$ genotype effect is stronger among females and in some cases in the opposite direction in males. ${ }^{35}$ In fact, in our fully adjusted model both gender and gender $\times$ genotype showed larger effects on depressive symptom severity than we detected for child abuse $\times$ genotype. Thus, further clinical trials, with particular attention to ancestry and gender, are required to determine the utility of 5HTTLPR genotype-guided psychosocial intervention in clinical practice.

The mechanism(s) by which the 5HTTLPR polymorphism confers differential susceptibility to the negative effects of child abuse is not clear. However, previous work has shown a link between child abuse and methylation in a CpG residue immediately upstream of the $5 H T T L P R^{36}$ and the $s$ allele appears to be more susceptible to methylation. ${ }^{37}$ Supporting this observation, a more recent study showed a decrease in SLC6A4 transcription in peripheral blood cells among $s$ allele carriers who reported a history of early trauma compared with $s$ allele carriers without early trauma and $l$ allele carriers with or without early trauma. ${ }^{38}$ Thus, the differential susceptibility conferred by the $s / s$ genotype that we and others have observed may in part be explained by DNA methylation mediated SLC6A4 transcription and possibly translation, although the data linking SLC6A4 methylation to its protein expression is limited. Further clinical as well as human post-mortem brain studies with accompanying ante-mortem data on child abuse/adverse life events are needed before firm conclusions on the mechanisms can be made.

The current study has several notable strengths absent in previous studies. We included comprehensive covariate adjustment, modelled both fixed and random effects, and used a longitudinal design. However, six key caveats should be acknowledged. First, depressive symptom severity was the primary outcome measure and as such results cannot be extrapolated to the categorical diagnosis of major depressive disorder. However, the use of quantitative traits and a dimensional perspective affords greater statistical power and the PHQ-9 has previously been validated as a replicable measure of symptom severity that correlates with decreasing physical and social functioning, increased disability days, and increased healthcare utilisation and thus is likely to be more meaningful compared with a dichotomous clinical diagnostic classification. ${ }^{14}$ Second, although all participants were of Northern/Western European ancestry, a strength in genetic association studies, we are not able to generalise these results to other ancestry groups, particularly given that frequencies of the $s$ and $l$ alleles are known to vary by ancestry. ${ }^{39}$ Third, detailed measures of depression treatment history were not available and adjustment of possible effects of antidepressant or psychotherapy treatment beyond the scope of self-reported use at baseline was not possible. Although we did not observe a difference in baseline antidepressant use by genotype, future follow-up studies should include detailed measurements of antidepressant use to evaluate whether the effect reported here is confounded by pharmacotherapy. Fourth, our linear mixed model approach required a large number of covariates and interactions to be examined in order to properly control for potential confounds. ${ }^{26}$ As a result, statistical power was likely suboptimal and our post hoc stratified analyses by genotype may have overestimated the true effects. Fifth, a priori we selected an additive genetic model but post hoc evaluation of our results suggests a recessive model $(s / s v . l$ carriers) may also fit our data. Although previous studies in the literature have almost exclusively assumed an additive model, future studies may benefit from examination of a recessive model. Last, this study did not examine all types of potential childhood adversity and relied on self-report. Childhood emotional abuse or neglect have also been associated with depressive symptoms in the primary care setting ${ }^{40}$ but were not queried and may have important effects not accounted for in this study. Furthermore, some have criticised the use of self-reported childhood abuse due to a number of biases (e.g. underreporting). ${ }^{41,42}$ However, it is likely that these biases would obscure the moderation effect observed, resulting in more conservative parameter estimates.

In summary, our results provide evidence that the 5HTTLPR $s / s$ genotype moderates the effects of severe childhood abuse on depressive symptom severity among adult primary care attendees. This effect was detected at baseline, persisted over the 5-year observation period and withstood robust adjustment of key confounders. If replicated, the 5HTTLPR polymorphism may serve as one of many candidate markers that could aid in identifying susceptibility to persistent depressive symptoms among individuals with a history of severe childhood abuse.

\section{Timothy B. Nguyen, BS, MD, Department of Psychiatry, The University of Melbourne Parkville, Victoria, Australia; Jane M. Gunn, PhD, MBBS, Department of General Practice, The University of Melbourne, Parkville, Victoria, Australia; Maria Potiriadis, BAppSc, GDip Clin Epi, M Prim HlthCare, Department of General Practice, The University of Melbourne, Parkville, Victoria, Australia; Ian P. Everall, BSC (Hons), MB ChB (Hons), PhD, DSC, FRCPsych, FRANZCP, FRCPath, Department of Psychiatry, The University of Melbourne, Parkville, Victoria, Australia, NorthWestern Mental Health, Melbourne, Victoria, Australia and Florey Institute of Neuroscience and Mental Health, The University of Melbourne, Parkville, Victoria, Australia; Chad A. Bousman, BS, MPH, PhD, Department of Psychiatry, The University of Melbourne, Parkville, Victoria, Australia, Department of General Practice, The University of Melbourne, Parkville, Victoria, Australia, Florey Institute of Neuroscience and Mental Health, The University of Melbourne, Parkville, Victoria, Australia and Centre for Human Psychopharmacology, Swinburne University of Technology, Hawthorne, Victoria, Australia}

Correspondence: Dr Chad Bousman, Melbourne Neuropsychiatry Centre, Department of Psychiatry, University of Melbourne, 161 Barry Street, Level 3, Carlton, VIC 3053, Australia. Email: cbousman@unimelb.edu.au

First received 12 Feb 2015, final revision 2 Jul 2015, accepted 19 Aug 2015

\section{Funding}

The diamond study is funded by the National Health and Medical Research Council (IDS 299869 454463, 566511 and 1002908) and the Victorian Centre for Excellence in Depression and Related Disorders, an initiative between beyondblue and the Victorian Government. The collection of DNA and genotyping was funded by the LEW Carty Chartable Fund (ID 7284). No funding body had a role in the study design; the collection, analysis and interpretation of data; or the writing of the manuscript for publication. We acknowledge the 30 dedicated general practitioners their patients and practice staff for making this research possible.

\section{Acknowledgements}

We thank the diamond project team, including associate investigators and researchers involved in the diamond study: Ms Aves Middleton, Ms KonstancjaDensley, Professor Helen Herrman, Professor Christopher Dowrick, Dr Gursharan Chana and casual research staff.

\section{References}

1 Arnow BA. Relationships between childhood maltreatment, adult health and psychiatric outcomes, and medical utilization. J Clin Psychiatry 2004; 65 (suppl 12): $10-5$

2 Chapman DP, Whitfield CL, Felitti VJ, Dube SR, Edwards VJ, Anda RF. Adverse childhood experiences and the risk of depressive disorders in adulthood. J Affect Disord 2004; 82: 217-25.

3 Mandelli L, Serretti A. Gene environment interaction studies in depression and suicidal behavior: an update. Neurosci Biobehav Rev 2013; 37: 2375-97. 
4 Heils A, Teufel A, Petri S, Stöber G, Riederer P, Bengel D, et al. Allelic variation of human serotonin transporter gene expression. J Neurochem 1996; 66: 2621-4.

5 Caspi A, Sugden K, Moffitt TE, Taylor A, Craig IW, Harrington H, et al. Influence of life stress on depression: moderation by a polymorphism in the 5-HTT gene. Science 2003; 301: 386-9.

6 Karg K, Burmeister M, Shedden K, Sen S. The serotonin transporter promoter variant (5-HTTLPR), stress, and depression meta-analysis revisited: evidence of genetic moderation. Arch Gen Psychiatry 2011; 68: 444-54.

7 Wankerl M, Wust S, Otte C. Current developments and controversies: does the serotonin transporter gene-linked polymorphic region (5-HTTLPR) modulate the association between stress and depression? Curr Opin Psychiatry 2010; 23 $582-7$.

8 Uher R. Gene-environment interaction: overcoming methodological challenges. Novartis Found Symp 2008; 293: 13-26; discussion 26-30, 68-70.

9 Keller MC. Gene $\times$ environment interaction studies have not properly controlled for potential confounders: the problem and the (simple) solution. Biol Psychiatry 2014; 75: 18-24.

10 Gunn JM, Gilchrist GP, Chondros P, Ramp M, Hegarty KL, Blashki GA, et al. Who is identified when screening for depression is undertaken in general practice? Baseline findings from the diagnosis, management and outcomes of depression in primary care (diamond) longitudinal study. Med J Aust 2008; 188: S119-25.

11 Radloff L. The CES-D scale: a self-report depression scale for research in the general population. Appl Psychol Meas 1977; 1: 385-401.

12 American Psychiatric Association. Task Force on DSM-IV. Diagnostic and Statistical Manual of Mental Disorders: DSM-IV-TR (4th edn). APA 2000, xxxvii, $943 p$

13 Spitzer RL, Kroenke K, Williams JB. Validation and utility of a self-report version of PRIME-MD: the PHQ primary care study. Primary care evaluation of mental disorders. Patient health questionnaire. JAMA 1999; 282: 1737-44.

14 Kroenke K, Spitzer RL, Williams JB. The PHQ-9: validity of a brief depression severity measure. J Gen Intern Med 2001; 16: 606-13.

15 MacMillan $\mathrm{HL}$, Fleming JE, Trocme $\mathrm{N}$, Boyle $\mathrm{MH}$, Wong $\mathrm{M}$, Racine $\mathrm{YA}$, et al. Prevalence of child physical and sexual abuse in the community. Results from the Ontario health supplement. JAMA 1997; 278: 131-5.

16 Straus MA. The conflict tactics scales and its critics: an evaluation and new data on validity and reliability. In Physical Violence in American Families: Risk Factors and Adaptations to Violence in 8,145 Families (eds MA Straus, RJ Gelles): 29-73. Transaction Publishers, 1990

17 Bagley C. Prevalence and correlates of unwanted sexual acts in childhood in national Canadian sample. Can J Public Health 1989; 80: 295-6.

18 Ware Jr J, Kosinski M, Keller SD. A 12-item short-form health survey: construction of scales and preliminary tests of reliability and validity. Med Care 1996; 34 220-33.

19 World Health Organization. The World Health Organization Quality of Life Assessment (WHOQOL): development and general psychometric properties. Soc Sci Med 1998; 46: 1569-85.

20 World Health Organization. Composite International Diagnostic Interview (CIDIAuto). 2.1 edition. WHO, 1997.

21 Norbeck JS. Modification of life event questionnaires for use with female respondents. Res Nurs Health 1984; 7: 61-71.

22 Enoch MA, Shen PH, Xu K, Hodgkinson C, Goldman D. Using ancestry-informative markers to define populations and detect population stratification. J Psychopharmacol 2006; 20: 19-26.

23 Gelernter J, Kranzler $H$, Coccaro $E F$, Siever $\sqcup$, New AS. Serotonin transporter protein gene polymorphism and personality measures in African American and European American subjects. Am J Psychiatry 1998; 155: 1332-8.
24 Wendland JR, Martin BJ, Kruse MR, Lesch KP, Murphy DL. Simultaneous genotyping of four functional loci of human SLC6A4, with a reappraisal of 5-HTTLPR and rs25531. Mol Psychiatry 2006; 11: 224-6.

25 Kraemer HC, Blasey CM. Centring in regression analyses: a strategy to prevent errors in statistical inference. Int J Methods Psychiatric Res 2004; 13: 141-51.

26 Keller MC. Gene $\times$ environment interaction studies have not properly controlled for potential confounders: the problem and the (simple) solution. Biol Psychiatry 2014: 75: 18-24.

27 West BT, Welch KB, Galecki AT. Linear Mixed Models: A Practical Guide Using Statistical Software. Chapman \& Hall/CRC, 2007

28 Labonte B, Suderman M, Maussion G, Navaro L, Yerko V, Mahar I. Genome-wide epigenetic regulation by early-life trauma. Arch Gen Psychiatry 2012; 69: 722-31.

29 Belsky J, Jonassaint C, Pluess M, Brummett B, Williams R. Vulnerability genes or plasticity genes? Mol Psychiatry 2009; 14: 746-54.

30 Eley TC, Sugden K, Corsico A, Gregory AM, Sham P, McGuffin P, et al. Geneenvironment interaction analysis of serotonin system markers with adolescent depression. Mol Psychiatry 2004; 9: 908-15.

31 Wilhelm $\mathrm{K}$, Mitchell PB, Niven $\mathrm{H}$, Finch A, Wedgwood L, Scimone A, et al. Life events, first depression onset and the serotonin transporter gene. Br J Psychiatry 2006; 188: 210-5.

32 Eley TC, Hudson JL, Creswell C, Tropeano M, Lester KJ, Cooper P, et al. Therapygenetics: the 5HTTLPR and response to psychological therapy. $\mathrm{Mol}$ Psychiatry 2012; 17: 236-7.

33 Bockting $\mathrm{CL}$, Mocking RJ, Lok A, Koeter MW, Schene AH. Therapygenetics: the 5 HTTLPR as a biomarker for response to psychological therapy? Mol Psychiatry 2013; 18: 744-5

34 Porcelli S, Fabbri C, Serretti A. Meta-analysis of serotonin transporter gene promoter polymorphism (5-HTTLPR) association with antidepressant efficacy. Eur Neuropsychopharmacol 2012; 22: 239-58.

35 Uher R, McGuffin P. The moderation by the serotonin transporter gene of environmental adversity in the aetiology of mental illness: review and methodological analysis. Mol Psychiatry 2008; 13: 131-46.

36 Vijayendran M, Beach SR, Plume JM, Brody GH, Philibert RA. Effects of genotype and child abuse on DNA methylation and gene expression at the serotonin transporter. Front Psychiatry 2012; 3: 55 .

37 Philibert R, Madan A, Andersen A, Cadoret R, Packer H, Sandhu H. Serotonin transporter mRNA levels are associated with the methylation of an upstream CDG island. Am J Med Genet B Neuropsychiatric Genet 2007; 144B: 101-5.

38 Wankerl M, Miller R, Kirschbaum C, Hennig J, Stalder T, Alexander N. Effects of genetic and early environmental risk factors for depression on serotonin transporter expression and methylation profiles. Transl Psychiatry 2014; 4: e402.

39 Gelernter J, Kranzler H, Cubells JF. Serotonin transporter protein (SLC6A4) allele and haplotype frequencies and linkage disequilibria in African- and EuropeanAmerican and Japanese populations and in alcohol-dependent subjects. Hum Genet 1997; 101: 243-6.

40 Spertus IL, Yehuda R, Wong CM, Halligan S, Seremetis SV. Childhood emotional abuse and neglect as predictors of psychological and physical symptoms in women presenting to a primary care practice. Child Abuse Negl 2003; 27 1247-58.

41 McKinney CM, Harris TR, Caetano R. Reliability of self-reported childhood physical abuse by adults and factors predictive of inconsistent reporting. Violence Vict 2009; 24: 653-68.

42 Hardt J, Rutter M. Validity of adult retrospective reports of adverse childhood experiences: review of the evidence. J Child Psychol Psychiatry 2004; 45: 260-73. 